\title{
Searching for flavor changing neutral currents at BESIII
}

\author{
Dayong Wang*广 \\ for the BESIII Collaboration
}

State Key Laboratory of Nuclear Physics and Technology, Peking University, Beijing, China

E-mail: dayong.wang@pku. edu.cn

The Flavor Changing Neutral Current decays(FCNC) is forbidden at tree level in the Standard Model (SM) and could only contribute through loops. Any direct observation beyond SM expectations could be a good probe of physics beyond SM. BESIII is a currently running tau-charm factory with the largest samples of on threshold charm meson pairs, directly produced charmonia and some other unique datasets at BEPCII collider. It has great potential to probe these FCNC decays from multiple channels. Here we review some of latest search results of FCNC decays from BESIII. We present the latest results of searches for the decay of $J / \psi \rightarrow D^{0} e^{+} e^{-}$, $\psi(3686) \rightarrow D^{0} e^{+} e^{-}, \psi(3686) \rightarrow \Lambda_{c}^{+} \bar{p} e^{+} e^{-}, D \rightarrow h\left(h^{\prime}\right) e^{+} e^{-}, D^{+} \rightarrow h^{+} e^{+} e^{-}$, etc. Other related searches, together with the prospects and challenges with other channels and the future data are also discussed.

The International Conference on High Energy Physics - ICHEP2018

4-11 July, 2018

Seoul, South Korea

\footnotetext{
* Speaker.

${ }^{\dagger}$ Partially supported by Joint Funds of the National Natural Science Foundation of China (Grants No. U1832207) and by Ministry of Science and Technology(Grants No. 2015CB856700).
} 


\section{Introduction}

BEPCII is the only currently running $\tau$-charm factory working at the c. m. energy range of 2.0-4.6GeV, located at IHEP, Beijing. This energy range has a lot of unique features which benefit greatly the rich physics programs. The BESIII detector has a geometrical acceptance of $93 \%$ of $4 \pi$ and consists a small-celled, helium-based main draft chamber, an electromagnetic calorimeter, a time-of-flight system for particle identification, a muon chamber system(MUC) made of about $1272 \mathrm{~m}^{2}$ of resistive plate chambers incorpared in the return iron of the superconducting solenoid with 1T magentic field. More details of the detector are described in Ref [1].

The BESIII experiment has accumulated 1.3B $J / \psi$ 's, $0.5 \mathrm{~B} \psi(3686)$ 's and $2.9 \mathrm{fb}^{-1}$ at $\psi(3773)$, all of which are the largest data sets in the world. The clean environments and high luminosity at BESIII provide ideal samples for searches for new physics(NP) beyond standard model.

The Flavor Changing Neutral Current decays(FCNC) is forbidden at tree level in the Standard Model (SM) due to the Glashow-Iliopoulos-Maiani mechanism [2], and could only contribute through loops. Any direct observation beyond SM expectations could be a good probe of physics beyond SM. BESIII has recently analysed several such FCNC decays, which are reviewed in this paper, including the searches for processes of $J / \psi \rightarrow D^{0} e^{+} e^{-}, \psi(3686) \rightarrow D^{0} e^{+} e^{-}$, $\psi(3686) \rightarrow \Lambda_{c}^{+} \bar{p} e^{+} e^{-}, D \rightarrow h\left(h^{\prime}\right) e^{+} e^{-}$, etc. Charge conjugation is always implied throughout.

\section{Search for $J / \psi \rightarrow D^{0} e^{+} e^{-}$and $\psi(3686) \rightarrow D^{0} e^{+} e^{-} \quad$ [3]}

This analysis is performed with $(1310.6 \pm 7.2) \times 10^{6} \mathrm{~J} / \psi$ events [4] and $(448.1 \pm 2.9) \times 10^{6}$ $\psi(3686)$ events [5], collected by BESIII. In the SM, the decay branching fraction for this kind of rare process is expected to be of order $10^{-10}$ to $10^{-13}$. Some NP models could have several of magnitudes higher decay rates, which could in the reach of BESIII.

The $D^{0}$ signals are reconstructed through its three prominent exclusive hadronic decay modes, $K^{+} \pi^{-}, K^{+} \pi^{-} \pi^{+}$, and $K^{+} \pi^{-} \pi^{+} \pi^{-}$, which have relatively large branching fractions, and suffer from relatively low background. After requring one $D^{0}$ and one pair of $e^{+} e^{-}$reconstructed, the distributions of the invariant masses from the three $D^{0}$ meson decay modes are simultaneously fit with unbinned maximum likelihood method for the $J / \psi$ and $\psi(3686)$ samples.

No $D^{0}$ signals are observed, and we compute the upper limits(UL) on the branching fraction at the $90 \%$ C.L. using a Bayesian method [6] with a flat prior,the correlated and un-correlated systematic uncertainties are incorporated. The results are $\mathscr{B}\left(J / \psi \rightarrow D^{0} e^{+} e^{-}\right)<8.5 \times 10^{-8}$ and $\mathscr{B}\left(\psi(3686) \rightarrow D^{0} e^{+} e^{-}\right)<1.4 \times 10^{-7}$, respectively. The limit on $\mathscr{B}\left(J / \psi \rightarrow D^{0} e^{+} e^{-}\right)$is more stringent by two orders in magnitude compared to the previous results, and the $\mathscr{B}(\psi(3686) \rightarrow$ $D^{0} e^{+} e^{-}$) is set for the first time.

\section{Search for $\psi(3686) \rightarrow \Lambda_{c}^{+} \bar{p} e^{+} e^{-}$[7]}

This analysis is performed with $(448.1 \pm 2.9) \times 10^{6} \psi(3686)$ events [5] collected by BESIII. The decay $\psi(3686) \rightarrow \Lambda_{c}^{+} \bar{p} e^{+} e^{-}$with $\Lambda_{c}^{+} \rightarrow p K^{-} \pi^{+}$is reconstructed with six charged tracks with zero net charge. The number of signal events is determined by examining the $\Lambda_{c}^{+}$signal in the $M_{p K^{-} \pi^{+}}$distribution, which is shown in Fig. 1(Left). 
No events survive within the signal region ranging from 2.25 to $2.32 \mathrm{GeV} / c^{2}$. The potential background in the signal region is estimated using events in the $M_{p K^{-} \pi^{+}}$sideband regions to be 1.5. We also estimate the number of background events to be zero using the inclusive MC sample and the data sample with $\sqrt{s}=3.773 \mathrm{GeV}$. As no candidate events are found in the signal region, the estimated number of background events is determined to be $0 \pm 1.5$ events. The upper limit on the $\mathrm{BF}(\mathscr{B})$ of the decay $\psi(3686) \rightarrow \Lambda_{c}^{+} \bar{p} e^{+} e^{-}+$c.c. is calculated to be $1.7 \times 10^{-6}$. The result is within the expectations of the SM, and no evidence for new physics is found.
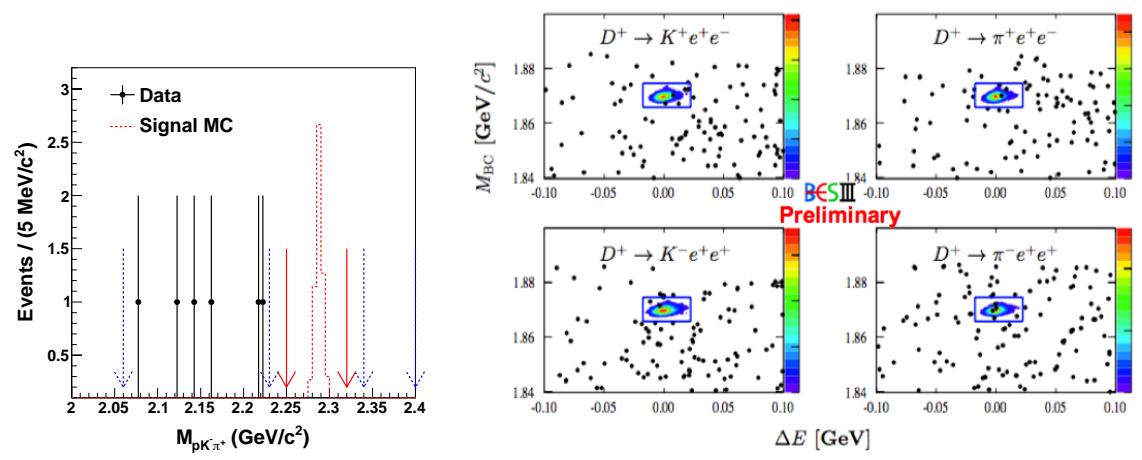

Figure 1: Left: Distribution of $M_{p K^{-} \pi^{+}}$for the data (dots with error bars) and signal MC sample (dashed histogram). Right: The scatter plots of $M_{\mathrm{BC}}$ versus $\Delta E$ of the accepted events in data, where the blue rectangle denotes the signal box, with the contour plots determined by MC simulation.

\section{Search for $D^{+} \rightarrow h^{+} e^{+} e^{-}$and $D^{+} \rightarrow h^{-} e^{+} e^{+}$}

Using an $e^{+} e^{-}$collision sample corresponding to an integrated luminosity of $2.93 \mathrm{fb}^{-1}$ [8] collected with the BESIII detector at $\sqrt{s}=3.773 \mathrm{GeV}$, we search for the FCNC decays $D^{+} \rightarrow$ $h^{+} e^{+} e^{-}$and the lepton-nubmer-violating decays $D^{+} \rightarrow h^{-} e^{+} e^{+}$, where $h$ are hadrons. The latter may be sensitive to NP models with Majorana neutrinos. Fig. 1 (Right) shows the resulting scatter plots of $M_{\mathrm{BC}}$ versus $\Delta E$ of the accepted events in data.

No signal excess is observed. As a result, we set the upper limits on the branching fractions for these decays at a $90 \% \mathrm{CL}$ as: $\mathscr{B}\left(D^{+} \rightarrow \pi^{+} e^{+} e^{-}\right)<0.3 \times 10^{-6}, \mathscr{B}\left(D^{+} \rightarrow K^{-} e^{+} e^{+}\right)<0.6 \times 10^{-6}$, $\mathscr{B}\left(D^{+} \rightarrow \pi^{-} e^{+} e^{+}\right)<1.2 \times 10^{-6}, \mathscr{B}\left(D^{+} \rightarrow K^{+} e^{+} e^{-}\right)<1.2 \times 10^{-6}$. Compared limits from previous experiments [6], the first two results are significantly improved than the previous restrictions and the other two limits are comparable with the world best results. These are BESIII preliminary results and formal publication will come later.

\section{Search for $D \rightarrow h\left(h^{\prime}\right) e^{+} e^{-}$[9]}

Using the same dataset at $\sqrt{s}=3.773 \mathrm{GeV}$, we also perform a search for the rare decays of $D \rightarrow h\left(h^{\prime}\right) e^{+} e^{-}$, where $h^{(\prime)}$ are hadrons. Double tagging(DT) method is used in the analysis. For each signal mode, $\Delta E_{\text {sig }}$ is required to be within $3 \sigma$ of the nominal value, and only the combination with the smallest $\left|\Delta E_{\text {sig }}\right|$ is kept. No significant excess over the expected backgrounds is observed in $M_{\mathrm{BC}}^{\mathrm{sig}}$ distributions of the surviving events. 
The ULs on the signal BFs at the $90 \% \mathrm{CL}$ are determined. The maximal signal significance is $2.6 \sigma$, for $D^{0} \rightarrow K^{-} \pi^{+} e^{+} e^{-}$. Its BF is expected to be dominated by the LD Bremsstrahlung and (virtual) resonance decay contributions in the lower and upper regions, so we divide the $M_{e^{+} e^{-}}$ distribution into three regions and determine the BFs in the individual regions.

Table 1: Results of the ULs on the BFs for the investigated rare decays of $D \rightarrow h\left(h^{\prime}\right) e^{+} e^{-}$at the $90 \% \mathrm{CL}$, and the corresponding results in the PDG.

\begin{tabular}{lcc||lcc} 
Signal decays & $\mathscr{B}\left(\times 10^{-5}\right)$ & PDG $[6]\left(\times 10^{-5}\right)$ & Signal decays & $\mathscr{B}\left(\times 10^{-5}\right)$ & PDG [6] $\left(\times 10^{-5}\right)$ \\
\hline$D^{+} \rightarrow \pi^{+} \pi^{0} e^{+} e^{-}$ & $<1.4$ & - & $D^{0} \rightarrow K^{-} K^{+} e^{+} e^{-}$ & $<1.1$ & $<31.5$ \\
$D^{+} \rightarrow K^{+} \pi^{0} e^{+} e^{-}$ & $<1.5$ & - & $D^{0} \rightarrow \pi^{+} \pi^{-} e^{+} e^{-}$ & $<0.7$ & $<37.3$ \\
$D^{+} \rightarrow K_{S}^{0} \pi^{+} e^{+} e^{-}$ & $<2.6$ & - & $D^{0} \rightarrow K^{-} \pi^{+} e^{+} e^{-\dagger}$ & $<4.1$ & $<38.5$ \\
$D^{+} \rightarrow K_{S}^{0} K^{+} e^{+} e^{-}$ & $<1.1$ & - & $D^{0} \rightarrow \pi^{0} e^{+} e^{-}$ & $<0.4$ & $<4.5$ \\
$\dagger$ in $M_{e^{+}} e^{-}$regions: & & - & $D^{0} \rightarrow \eta e^{+} e^{-}$ & $<0.3$ & $<11$ \\
{$[0.00,0.20) \mathrm{GeV} / c^{2}$} & $<3.0\left(1.5_{-0.9}^{+1.0}\right)$ & - & $D^{0} \rightarrow \omega e^{+} e^{-}$ & $<0.6$ & $<18$ \\
{$[0.20,0.65) \mathrm{GeV} / c^{2}$} & $<0.7$ & $D^{0} \rightarrow K_{S}^{0} e^{+} e^{-}$ & $<1.2$ & $<11$ \\
{$[0.65,0.90] \mathrm{GeV} / c^{2}$} & $<1.9\left(1.0_{-0.4}^{+0.5}\right)$ & - & & & \\
\hline
\end{tabular}

All these results are listed in Table 1, and are all within the SM predictions. For the four-body $D^{+}$decays, the searches are performed for the first time. The reported ULs of the $D^{0}$ decays are improved in general by a factor of 10, compared to previous measurements [6]. All the measured ULs on the BFs are above the SM predictions, which include both LD and SD contributions.

\section{Summary and outlook}

BESIII has performed a series of searches for FCNC processes. Though most of the upper limits are larger than the SM predictions, they may help to discriminate the different new physics models or to constrain the parameters in the different physics models. Additionally, higher statistics $J / \psi, \psi(3686)$ and D meason samples may help to improve the sensitivity of the measurements. BESIII efforts with more related channels and more coming data will be continued to further test the standard model with higher precision in future.

\section{References}

[1] M. Ablikim et al. (The BESIII Collaboration), Nucl. Instrum. Meth. A 614, 345 (2010).

[2] S. L. Glashow, J. Iliopoulos and L. Maiani, Phys. Rev. D 2, 1285 (1970).

[3] M. Ablikim et al. (The BESIII Collaboration), Phys. Rev. D 96, 111101 (2017).

[4] M. Ablikim et al. (The BESIII Collaboration), Chin. Phys. C 36, 915 (2012); M. Ablikim et al. (The BESIII Collaboration), Chin. Phys. C 41, 013001 (2017).

[5] M. Ablikim et al. (The BESIII Collaboration), Chin. Phys. C 37, 063001 (2013); M. Ablikim et al. (The BESIII Collaboration), Chin. Phys. C 42, 023001 (2018).

[6] C. Patrignani et al. (Particle Data Group), Chin. Phys. C 40, 100001 (2016) and 2017 update.

[7] M. Ablikim et al. (The BESIII Collaboration), Phys. Rev. D 97, 091102 (2018).

[8] M. Ablikim et al. (BESIII Collaboration), Chin. Phys. C 37, 123001 (2013); M. Ablikim et al. (BESIII Collaboration), Phys. Lett. B 753, 629 (2016).

[9] M. Ablikim et al. (BESIII Collaboration), Phys. Rev. D 97, 072015 (2018). 